\title{
Time-Dependent Ginzburg-Landau Simulations of Vortex Guidance by Twin Boundaries*
}

\author{
G. W. Crabtree ${ }^{\text {l }}$, G. K. Leaf ${ }^{2}$, H. G. Kaper ${ }^{2}$, V. M. Vinokur ${ }^{1}$, A. E. Koshelev ${ }^{1}$, \\ D. W. Braun ${ }^{2}$, D. M. Levine ${ }^{2}$, W. K. Kwok ${ }^{1}$, and J. A. Fendrich ${ }^{1}$ \\ ${ }^{1}$ Materials Science Division \\ ${ }^{2}$ Mathematics and Computer Science Division \\ Argonne National Laboratory, Argonne, IL 60439, USA
}
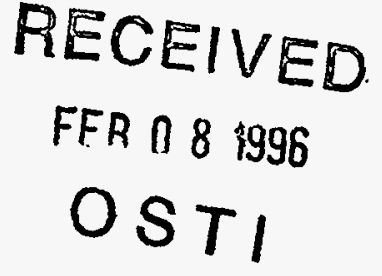

\begin{abstract}
The driven motion of vortices in the presence of a planar defect is simulated by means of the timedependent Ginzburg-Landau equations. Guided motion of the vortices, both internal and external to the twin boundary, is found to occur over a range of driving forces. Experimental transport data on a single crystal of $\mathrm{YBa}_{2} \mathrm{Cu}_{3} \mathrm{O}_{7}$ are consistent with guided motion.
\end{abstract}

\section{INTRODUCTION}

Twin boundaries in the high-temperature superconductor $\mathrm{YBa}_{2} \mathrm{Cu}_{3} \mathrm{O}_{7}$ provide a prototypical example of strong anisotropic pinning by an extended defect. Strong pinning by twin boundaries was first suggested [1] shortly after their existence was recognized, and the high degree of anisotropy of their pinning properties has since been characterized in transport [2], torque magnetometry [3], decoration [4,5] and magnetization [6,7] experiments. Subsequent magnetooptical experiments [8-13] led to conflicting interpretations of vortex pinning at twin boundaries. While initial experiments [8,9] showed that twin boundaries were planes of reduced pinning, allowing flux to penetrate more deeply into the superconductor than in the surrounding untwinned regions of the crystal, later studies [10] found twin boundaries to be barriers to flux penetration. The apparent conflict was resolved by further magneto-optical experiments [11-13], which revealed that the nature of the twin boundary pinning depends on the direction of the Lorentz force driving the vortex motion. Barrier action occurs when the Lorentz force is perpendicular to the twin boundary, while deep penetration occurs when the Lorentz force is parallel to the boundary. The combination of two very different pinning behaviors in the same defect and the dramatic dependence of the pinning behavior on vortex motion make twin boundaries an ideal laboratory for the exploration of static and dynamic aspects of pinning by extended defects.

The magneto-optical experiments suggested that guidance of vortices by a twin boundary occurs when the vortex velocity is at an intermediate angle to the boundary plane. Two mechanisms for such guidance can be imagined. In the first, vortices travel within the twin boundary, channeled by the deep pinning potential wells separating the interior of the boundary from the adjoining bulk superconductor. Here it is assumed that the boundary has a finite width and that one or more vortices can travel along its length. This "penetration effect" appears in the experiment as a channel of relatively deep flux penetration along the twin boundary. In the second mechanism, the vortices are imagined to be pinned and stationary on the twin boundary. Vortex-vortex repulsion prevents nearby vortices from crossing the twin boundary, which effectively forms a barrier to perpendicular motion. Parallel motion is allowed, so that driving forces at intermediate angles produce motion only along the boundary. This kind of "external guidance" is to be distinguished from internal guidance, as the motion takes place outside and adjacent to the boundary.

To further explore the nature of pinning by twin boundaries and the mechanisms of planar guidance under dynamic conditions, we carried out large-scale simulations of vortex motion by solving the time-dependent Ginzburg-Landau equations with a driving force and in the presence of planar pinning defects. To explore the collective aspects of the motion, a statistically significant number of vortices must be followed. Such large-scale simulations are beyond the scope of most computer systems, which typically limit the simulation of the time-dependent Ginzburg-Landau equations to tens of vortices. Our simulations were carried out on Argonne National Laboratory's IBM Scalable POWERparallel (SP) system, a 128-processor parallel architecture, each processor being an IBM RS/6000 with 128 Mbytes of memory, and typically accounted for hundreds of vortices. Most of the simulations were done with 16 processors running simultaneously and required close to 100 hours to produce a detailed record of the dynamic evolution of the system over a finite time interval. The results show both external and internal guidance occurring at

\footnotetext{
- Proceedings of the International Symposium on Frontiers of High $T_{c}$ Superconductivity, October 27-29, 1995, Morioka, Japan, Physica C 
different driving forces and penetration of the twin boundary barrier at high driving force. Experimental transport data on a twinned single crystal of $\mathrm{YBa}_{2} \mathrm{Cu}_{3} \mathrm{O}_{7}$ with the same geometry as in the simulation provide evidence for vortex guidance and penetration at intermediate and high driving forces.

\section{SIMULATION}

The time-dependent Ginzburg-Landau equations used in the simulation are those first proposed by Schmid [14] and later analyzed by Gor'kov and Eliashberg [15]:

$$
\frac{\partial \psi}{\partial t}=-\frac{\delta \mathcal{L}}{\delta \psi^{*}} \quad \sigma \frac{\partial \mathbf{A}}{\partial t}=-\frac{\delta \mathcal{L}}{\delta \mathbf{A}}-\nabla \times \nabla \times \mathbf{A},
$$

where $\mathcal{L}$ is the Helmholtz free energy functional

$$
\mathcal{L}=a|\psi|^{2}+\frac{b}{2}|\psi|^{4}+\frac{1}{2 m_{s}}\left|\left(\frac{\hbar}{i} \nabla-\frac{e_{s}}{c} \mathbf{A}\right)\right|^{2}
$$

The other symbols have their usual meaning. We work in the zero-potential gauge, $\Phi=0$, using link variables to discretize the fields. Details of the computational method can be found elsewhere [16].

The simulated system consisted of a superconducting planar slab, with a mutually perpendicular current and applied magnetic field in the plane of the slab. Along the field direction the slab was infinite and homogeneous, so that the problem became two-dimensional. Along the current direction a periodic boundary condition was used to insure continuity of the current without complicated end effects at the entrance and exit surfaces. The repeat distance of the periodic boundary condition was $48 \lambda$ ( $\lambda$ is the magnetic penetration depth). The current and field produce a Lorentz force in the direction perpendicular to both. Along this direction the sample had a finite width of $32 \lambda$ and a physical boundary represented by the condition $\mathbf{J} \cdot \mathbf{n}=0$, where $\mathbf{J}$ is the total current inside the sample and $\mathbf{n}$ is normal to the surface. A single twin boundary was inserted into the sample in the form of a planar slab of thickness $2.0 \xi$ ( $\xi$ is the coherence length), oriented parallel to the field and $45^{\circ}$ to the current direction. With this orientation, the plane of the boundary is $45^{\circ}$ from the Lorentz force direction, a geometry commonly encountered in experiments. The pinning of the twin boundary was modeled by a local reduction of the condensation energy in the interior of the slab. To provide the experimentally observed resistance to vortex motion within the twin boundary plane [2], the reduction was allowed to fluctuate randomly with a Gaussian distribution around a mean value of $56 \%$ and a standard deviation of $25 \%$ of the bulk value. Outside the twin boundary, there was no pinning in the bulk of the sample. This model provides an anisotropic pinning configuration with two pinning energy scales: one for motion parallel to the twin boundary, determined by the fluctuations in the condensation energy, and another for motion transverse to the twin boundary, determined by the reduction in condensation energy from the bulk value. The Ginzburg-Landau parameter $\kappa=\lambda / \xi=4$ for the simulation. No temperature fluctuations were included, so that the simulations reflect the solid rather than the liquid vortex state.

Each simulation was initiated by a sequence of steps. First a small magnetic field was applied to bring the superconductor into the Meissner state. Then a large field, equivalent to $\sim 77 \%$ of the thermodynamic critical field, was applied to bring the system into the vortex state. Simultaneously, a transport current was applied to the system by imposing an applied field difference across the two physical boundaries. By Ampere's law, the field difference $\Delta H$ causes a current $I=(c / 4 \pi) \Delta H$ to flow in the sample. Because only the boundary fields are specified, no restriction is put on the transport current distribution within the sample. The transport current induces a Lorentz force on the vortices, which sets them in motion. The motion is allowed to evolve for $1.8 \times 10^{6}$ time steps, well after the system reaches steady state. The data used to analyze the vortex motion were collected over the next $0.6 \times 10^{6}$ time steps in the steady state. Three currents in the ratio 1:2:4 were applied in separate simulations to probe the effect of an increased driving force on the dynamics. The largest current had a value $\sim 8 \%$ of the depairing current.

\section{RESULTS}

Figure 1 shows the results of the simulation at weak current. (In this and the following figures, the magnetic field direction is out of the page, the transport current direction is vertically up, and the Lorentz force direction is horizontal to the right.) To show the motion of the vortices, the positions of all the vortices sampled during the steady-state motion are plotted in the same frame. Stationary vortices appear as points, moving vortices as lines whose length

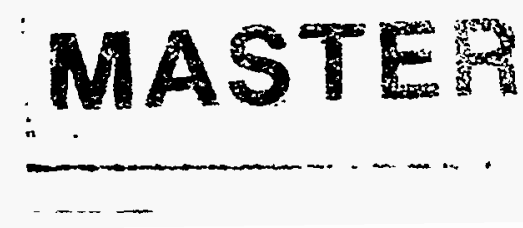


indicates their average velocity. The position of the twin boundary is marked by the diagonal row of stationary vortices extending from the upper right corner. The Lorentz force induced by the weak current is insufficient to overcome the random pinning forces in the twin boundary, and it effectively forms an impenetrable barrier to vortex motion. The vortices on either side of the boundary move in the parallel direction, displaying the kind of external guidance expected for a barrier geometry.

There are interesting features about the guided motion that reflect the special dynamics of vortices in the solid rather than the liquid state. The velocity of the vortices changes abruptly from zero on the twin boundary to approximately its largest value in one vortex spacing on either side of the twin boundary. At larger distances from the twin boundary, the vortex velocity remains approximately constant or decreases. This is different from what is expected for a liquid, where the velocity profile would be expected to grow slowly and monotonically from zero at the boundary to its largest value deep in the liquid. Furthermore, the velocities for several rows above the twin boundary are nearly equal, then fall suddenly almost to zero in one vortex spacing. Likewise, below the twin boundary, the velocities are nearly equal for two rows, then fall to zero. Further below the twin boundary, the velocity rises again, and again falls abruptly to zero.

Both the velocity discontinuities and the high correlation of neighboring velocities are characteristic of the motion of a solid. These two features arise from the shear elastic energy of the solid, which imposes an elastic energy cost on relative displacements of neighboring vortex positions away from their equilibrium values. Because of the shear elastic energy, neighboring vortices tend to maintain the relative positions of the perfect lattice. Thus, sections of the vortex system tend to move with approximately the same velocity. When velocity differences must be accommodated, they are compressed into one vortex spacing as discontinuous changes, because this minimizes the elastic energy of the neighboring sections. In contrast, velocity changes in liquids are accommodated continuously, because there is no shear elastic energy cost discouraging relative displacements of neighboring vortices. The high correlation of the velocities of neighboring vortices and the accommodation of velocity changes as discontinuities are characteristic features of plastic flow in solids.

The simulations reveal the microscopic origins of some of the plastic flow discontinuities in the velocity. The surface barrier arising from the boundary condition $\mathbf{J} \cdot \mathbf{n}=0$ at the left and right edges has a strong effect on the motion of the vortices. In a sample without a twin boundary, the surface barrier is homogenous and the critical current required to overcome the barrier can be computed theoretically. Such estimates of this critical current are confirmed by simulations without twin boundaries [17]. The presence of the twin boundary introduces a weak region in the surface barrier where it intersects with the edge of the sample, allowing vortices to enter at lower current and influencing their entry pattern, as noted below. Examination of the detailed time evolution of the system shows that the motion exhibited in Figure 1 does not occur continuously, but as a series of rearrangements prompted by the entry of vortices from the left. Each entering vortex distorts the vortex arrangement near its entry point, and long range relaxation occurs according to the pattern indicated in Figure 1. Thus, vortices entering above the twin boundary trigger the motion of the four rows just above the boundary, while those entering just below the boundary initiate the motion below. The boundary itself acts as a gate, providing a region of weak condensation energy at the left edge of the sample where vortices prefer to enter the system. This gate effect is most obvious for vortices entering the sample immediately below the boundary. It is further illustrated by comparison with simulations containing no twin boundary, where the number of vortices in the system is smaller and the surface barrier prevents vortex entry in steady state at this current [17]. While the entry of vortices triggers some of the motion in Figure 1, there is additional motion which occurs far from both the twin boundary and the sample edge. Two rows of vortices near the bottom of the frame move parallel to the boundary without any direct connection to entering vortices or to the boundary itself. This motion illustrates the long-range collective nature of the plastic flow response of the system.

Figure 2 shows the vortex trajectories at intermediate current. Here the pattern of motion is quite different from that at weak current. Many of the vortices break through the twin boundary, the Lorentz force being large enough to overcome the pinning forces of some of the pinning sites. The Lorentz force does not dominate all the pinning sites, as shown by the one remaining stationary vortex in the lower left part of the boundary. This rough balance between the pinning and Lorentz forces leads to a qualitatively new behavior. The external guidance, which was dominant at weak current, is missing at intermediate current, although a clear vestige of it can still be seen below the lower left section of the boundary. Elsewhere along the boundary, weak external guidance can be seen, extending no more than one row on either side of the boundary.

Inside the boundary, there is considerable motion along the boundary in certain regions, especially at the lower left and upper right. This is internal guidance. It differs from the penetration effect suggested by the magneto-optical experiments, in that it occurs in the absence of any bulk pining outside the twin boundary. Thus, the vortex density inside the boundary is not substantially different from that outside the boundary. In the magneto-optical experiments, the twin boundaries were seen to admit flux when the bulk of the sample was relatively flux free. To study such a 
penetration effect, the simulation would have to take into account bulk pinning outside the twin boundary. Figure 2 shows that the internal motion is associated with external motion of the neighboring vortices on either side of the boundary. This behavior is consistent with the influence of the shear modulus, which tends to cause neighboring vortices to move at the same velocity. Thus, coupled internal and external motion is a natural consequence of a finite shear modulus.

The internal motion can be understood physically in terms of the random pinning potential and the balance between the Lorentz and pining forces. The linear array of random pinning wells creates a highly anisotropic pinning environment. A vortex on a particular pinning site sees the full well potential as the barrier to motion in the direction perpendicular to the boundary. In contrast, in the parallel direction the potential barrier to the next well may be greatly reduced, if the neighboring well has approximately the same depth. Thus it may be much easier for the vortex to jump to the neighboring well than to the bulk of the superconductor. This kind of internal motion will stop if the moving vortex encounters a particularly well pinned vortex, as occurs near the lower left of the boundary, or if the well depth becomes shallow enough for the vortex to jump to the bulk. Such escapes to the bulk can be seen in the upper right section of the boundary.

The effect of the boundary on the vortex motion at intermediate current is apparent, but it is not the dominant force as was the case at weak current. This is clearly seen in the parts of the sample away from the boundary, where the vortices move steadily to the right, rather than diagonally as at weak current where the boundary was an effective barrier. The spatial range of influence of the boundary is confined to one vortex spacing. Beyond that, there is at most a small influence on the vortex velocity directions, in contrast to the case at weak current where the barrier was impenetrable. Ultimately, most of the vortices penetrate the boundary after undergoing a perturbation in velocity direction at the boundary itself. Thus the guidance provided by the boundary is local in nature and not a determining feature of the average motion.

Figure 3 shows the vortex trajectories at strong current. Only the trajectories in the first $20 \%$ of the steady-state time window are shown, because otherwise the frame becomes too crowded to resolve individual vortex paths. Here the boundary is nearly powerless against the Lorentz force, and many of the vortices stream through with hardly a change in direction. There is a hint of internal guidance at the extreme lower left of the boundary, but otherwise the trajectories do not show coherent vortex motion that can be attributed to the boundary.

The phenomenon of twin boundary guidance may provide a possible explanation of recent transport data on twinned samples. Here we present data on a single crystal containing only two twin boundaries, 140 microns apart, oriented at $45^{\circ}$ to the current direction. The current was applied in the ab plane, with the field along the $c$ axis, inducing a Lorentz force at $45^{\circ}$ to the twin boundary. Figure 4 shows the resistivity as a function of field at a fixed temperature of $90.61 \mathrm{~K}$ for several current levels. The sharp rise in resistivity above $0.55 \mathrm{~T}$ at low current $\left(13.5 \mathrm{~A} / \mathrm{cm}^{2}\right)$ is due to the melting transition, as explained elsewhere [18] in detail for this crystal. Below $0.55 \mathrm{~T}$, the vortex system is in the solid state. At $13.5 \mathrm{~A} / \mathrm{cm}^{2}$, the pinning forces are strong enough to immobilize the solid. At higher current, the vortex solid begins to move, producing significant dissipation in the range $\sim 0.35-0.5 \mathrm{~T}$. Remarkably, the dissipation is always lowest just below the melting transition, a manifestation of the "pre-melting peak effect" [18].

In the region of significant dissipation at intermediate current, $40 \mathrm{~A} / \mathrm{cm}^{2}$, there are two branches to the resistivity curve, one at about $0.3 \mu \Omega-\mathrm{cm}$ and another at about $0.15 \mu \Omega$-cm. Each branch is clearly defined, well outside the noise levels in the experiment. Field sweeps up and down find the two branches at the same resistance levels, though not at the same field positions. The behavior is as if a switching phenomenon were operating, choosing between two dynamic configurations with different average vortex velocities transverse to the current. Such a situation would arise if the vortices were guided by the twin boundaries on the lower branch, but broke through the twin boundaries on the upper branch. The expected ratio of the two resistivities can be estimated under the assumption that the vortex velocity scales with the Lorentz force, as is the case for viscous motion. Then the velocity along the twin boundary in the guided case will respond to the component of the Lorentz force in that direction, $F_{L} / \sqrt{2}$. The measured resistivity is sensitive only to the component of the velocity transverse to the current, $F_{L} /(\sqrt{2})^{2}=F_{L} / 2$. If the vortices break through the boundary, their velocity responds to the full Lorentz force, $F_{L}$. Thus, the two resistivities scale by a factor of 2 , as observed in the experiment. At higher current, where the Lorentz force overcomes the twin boundary pinning, there is only one branch to the resistivity curve. This behavior as a function of current is consistent with the phenomenon of twin boundary guidance and penetration as observed in the simulations.

\section{SUMMARY}

We have carried out large-scale simulations of the driven motion of vortices in the presence of a planar defect like a twin boundary in $\mathrm{YBa}_{2} \mathrm{Cu}_{3} \mathrm{O}_{7}$, using the fully nonlinear time-dependent Ginzburg-Landau equations. In order to 
follow the motion of a statistically significant number of vortices, the simulations required substantial computing power. On 16 processors of Argonne National Laboratory's IBM SP system, each of the simulations required close to 100 hours. Highly organized motion of the vortices was found at weak Lorentz driving force. The vortices external to the twin boundary were constrained to move parallel to the boundary, while those internal to the boundary were immobilized, forming an impenetrable barrier to motion across the boundary. At intermediate driving force, where the driving and pinning forces were comparable, parallel motion of vortices internal to the boundary occurred, coupled with similar motion of neighboring vortices external to the boundary. In other parts of the boundary, the vortices penetrated routinely. The guiding influence of the boundary was limited to approximately one vortex spacing on either side, much less than in the weak driving force case. At high driving force, the vortices freely penetrated the boundary with only a slight perturbation in their velocity directions, and there was no indication of correlated guidance. Transport data on a single crystal of $\mathrm{YBa}_{2} \mathrm{Cu}_{3} \mathrm{O}_{7}$ with two twin boundaries in the same orientation as in the simulations show resistivity patterns which are consistent with guidance and penetration.

We thank Ulrich Welp for useful and stimulating conversations. This work was supported by the U.S. Department of Energy under contract \#W-31-109-ENG-38, through the Office of Basic Energy Sciences-Materials Science (GWC, VMV, WKK) and the Office of Computational and Technology Research-Mathematical, Information, and Computational Sciences Division subprogram (GKL, HGK, DWB, DML), and by the U. S. National Science Foundation Science and Technology Center for Superconductivity under contract \#DMR 91-20000 (AEK, JAF).

[1] G. W. Crabtree, J. Z. Liu, A. Umezawa, W. K. Kwok, C. H. Sowers, S. K. Malik, B. W. Veal, D. J. Lam, M. B. Brodsky, and J. W. Downey, Phys Rev B 36, 4021 (1987).

[2] W. K. Kwok, U. Welp, G. W. Crabtree, K. G. Vandervoort, R. Hulscher, J. Z. Liu, Phys Rev Letters 64, 966 (1990).

[3] E. M. Gyorgy, R. B. van Dover, L. F. Schneemeyer, A. E. White, H. M. O'Bryan, R. J. Felder, J. V. Waszczak, and W. W. Rhodes, Appl Phys Letters 56, 2465 (1990).

[4] L. Ya Vinnikov, L. A. Gurevich, G. A. Emel'chenko, and Yu. A. Ossip'yan, Pis'ma Zh Eksp. Teor. Fiz. 47, 109 (1988) [JETP Letters 47, 131 (1988)]; Solid State Commun 67, 421 (1988).

[5] G. J. Dolan, G. V. Chandrashekhar, T. R. Dinger, C. Feild, and F. Holtzberg, Phys Rev Letters 62, 827 (1989).

[6] L. J. Swartzendruber, A. Roitburd, D. L. Kaiser, F. W. Gayle, and L. H. Bennett, Phys Rev Letters 64, 483 (1990).

[7] U. Welp, W. K. Kwok, G. W. Crabtree, K. G. Vandervoort, J. Z. Liu, Appl Phys Letters 57,84 (1990).

[8] C. A. Duran, P. L. Gammel, R. Wolfe, V. J. Fratello, D. J. Bishop, J. P. Rice, D. M. Ginsberg, Nature (London) 357,474 (1992).

[9] M. Turchinskaya, D. L. Kaiser, F. W. Gayle, A. J. Shapiro, A. Roitburd, V. Vlasko-Vlasov, A. Polyanskii, and V. Nikitenko, Physica C 216, 205 (1993).

[10] V. K. Vlasko-Vlasov, L. A. Dorosinskii, A. A. Polyanskii, V. I. Nikitenko, U. Welp, B. W. Veal, and G. W. Crabtree, Phys Rev Letters 72, 3246 (1994).

[11] U. Welp, T. Gardiner, D. Gunter, J. Fendrich, G. W. Crabtree, V. K. Vlasko-Vlasov, V. I. Nikitenko, Physica C 235-240, 241-244 (1994).

[12] C. A. Duran, P. L. Gammel, D. J. Bishop, J. P. Rice, D. M. Ginsberg, Phys Rev Letters 74, 3712 (1995).

[13] U. Welp, T. Gardiner, D. O. Gunter, B. W. Veal, G. W. Crabtree, V. K. Vlasko-Vlasov, V. I. Nikitenko, Phys Rev Letters 74, 3713, (1995).

[14] A. Schmid, Phys. Kondens. Materie 5, 302 (1966).

[15] L. P. Gor'kov and G. M. Eliashberg, Sov Phys JETP 28, 1991 (1969).

[16] W. D. Gropp, H. G. Kaper, G. K. Leaf, D. M. Levine, M. Palumbo, and V. M. Vinokur, J. Comp. Phys. (in press).

[17] D. W. Braun, G. W. Crabtree, H. G. Kaper, A. E. Koshelev, G. K. Leaf, D. M. Levine, V. M. Vinokur, preprint MCSP537-0895, Argonne National Laboratory.

[18] W. K. Kwok, J. A. Fendrich, C. J. van der Beek, G. W. Crabtree, Phys Rev Letters 73, 2614 (1994).

Fig. 1. Vortex trajectories near a twin boundary at weak transport current. The magnetic field is out of the page, the current direction is vertically upward, and the Lorentz force direction is horizontally to the right. The position of the twin boundary is marked by the line of stationary vortices extending diagonally from the upper right.

Fig. 2. Vortex trajectories at intermediate transport current. 
Fig. 3. Partial vortex trajectories at strong transport current.

Fig. 4. Resistivity as a function of magnetic field at fixed temperature for a single crystal of $\mathrm{YBa}_{2} \mathrm{Cu}_{3} \mathrm{O}_{7}$ containing two twin boundaries 140 microns apart, oriented $45^{\circ}$ from the current direction. The current is in the ab plane, with field along the $c$ axis. Three currents are shown. The arrows at intermediate current indicate up and down field sweeps. Switching behavior occurs for the intermediate current, consistent with vortex guidance and penetration at the twin boundaries.

\section{DISCLAIMER}

This report was prepared as an account of work sponsored by an agency of the United States Government. Neither the United States Government nor any agency thereof, nor any of their employees, makes any warranty, express or implied, or assumes any legal liability or responsibility for the accuracy, completeness, or usefulness of any information, apparatus, product, or process disclosed, or represents that its use would not infringe privately owned rights. Reference herein to any specific commercial product, process, or service by trade name, trademark, manufacturer, or otherwise does not necessarily constitute or imply its endorsement, recommendation, or favoring by the United States Government or any agency thereof. The views and opinions of authors expressed herein do not necessarily state or reflect those of the United States Government or any agency thereof. 


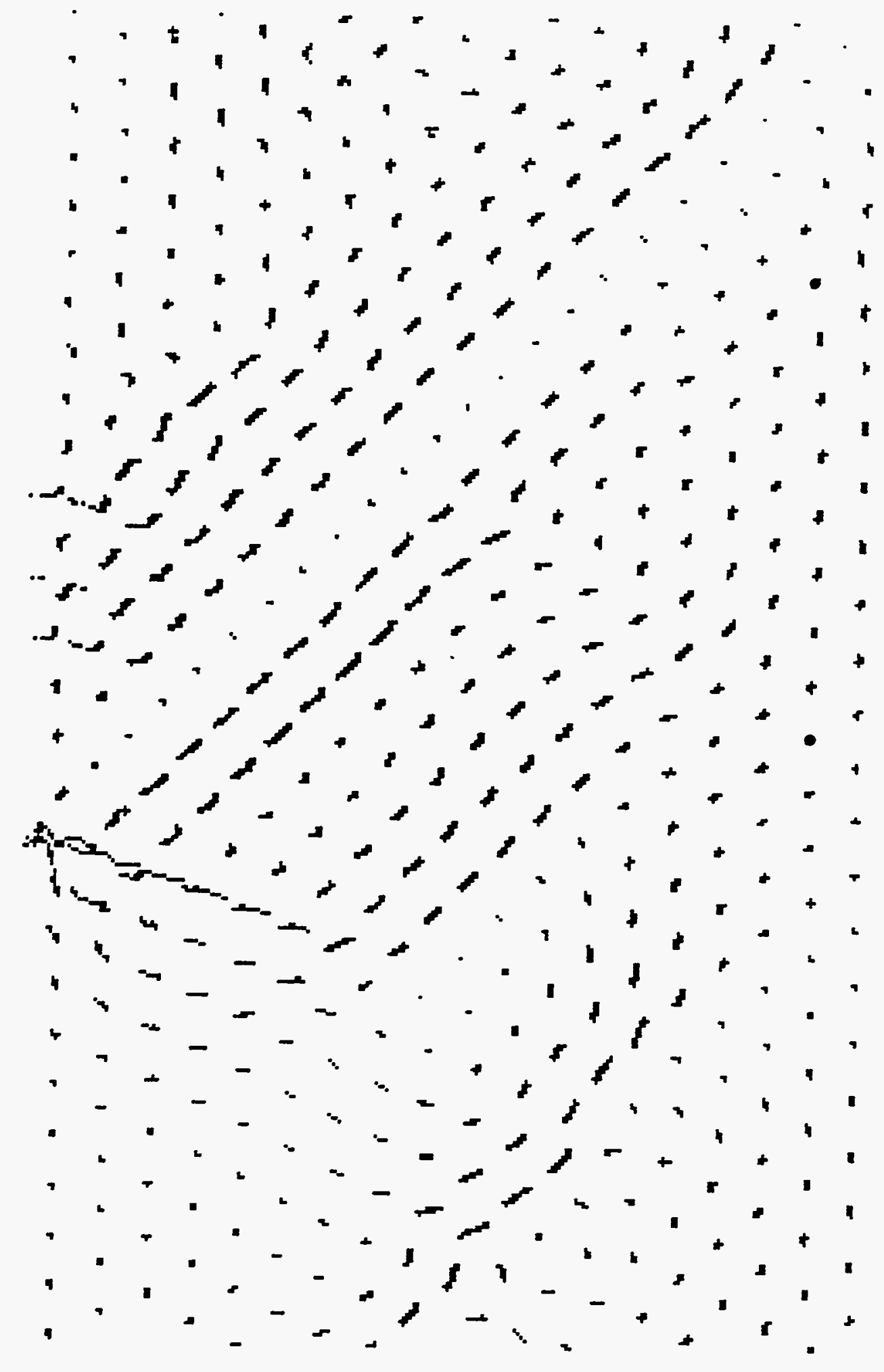

Figure 1 
: " : S م س : :

. ix F"

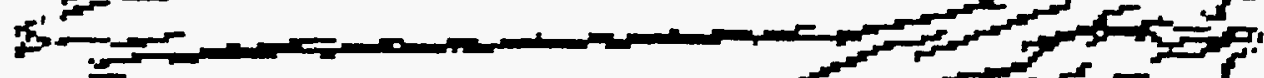
(1) $=2$ F .

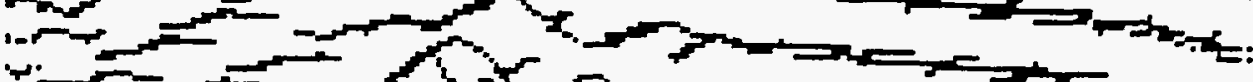

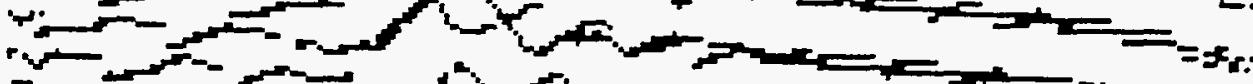
r of $=5$ $\rightarrow$ F $r=2=0$

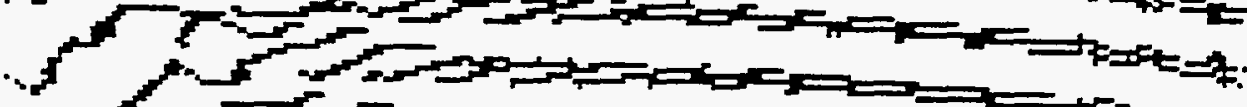
: . 


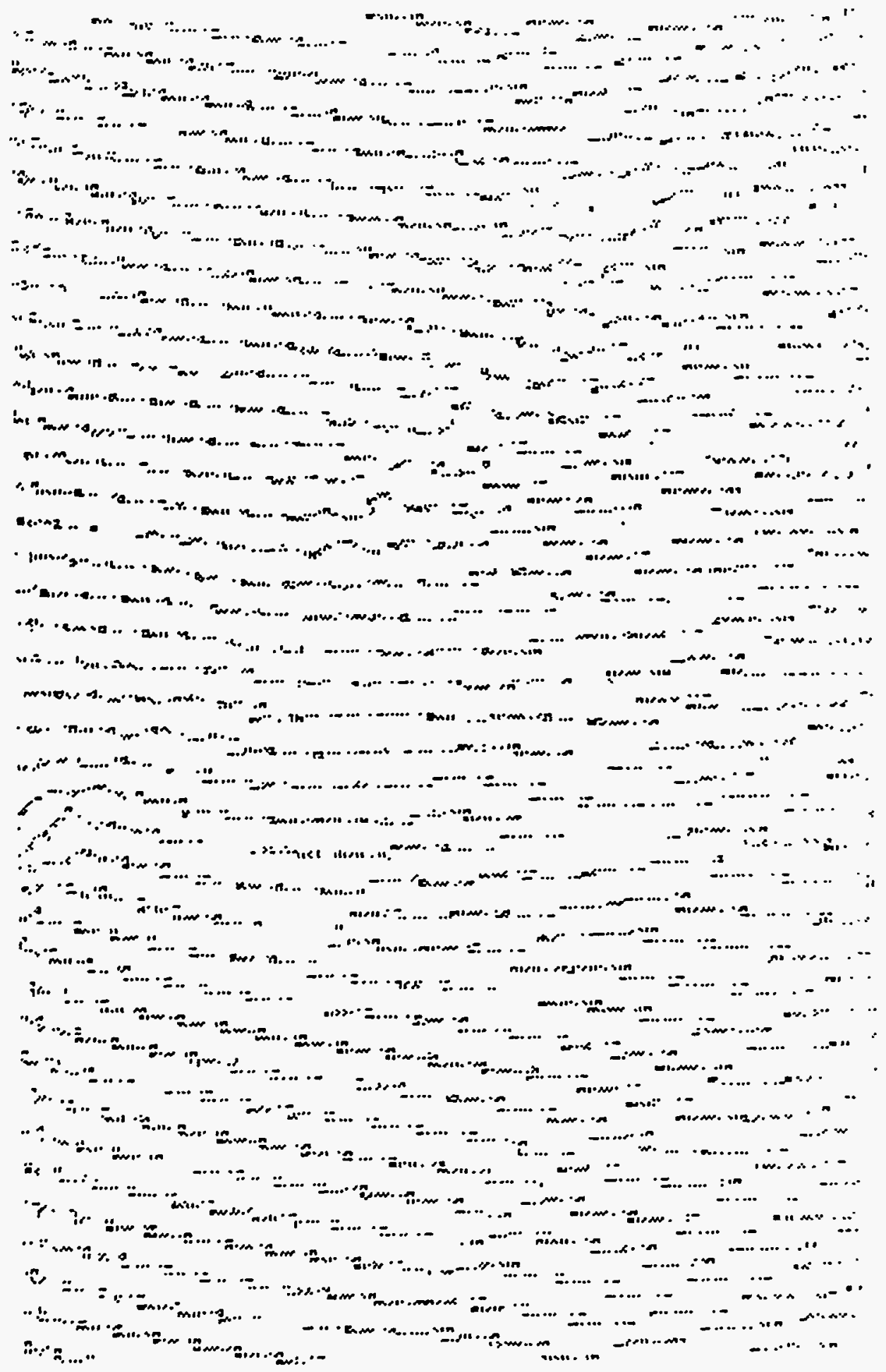

Figure 3 


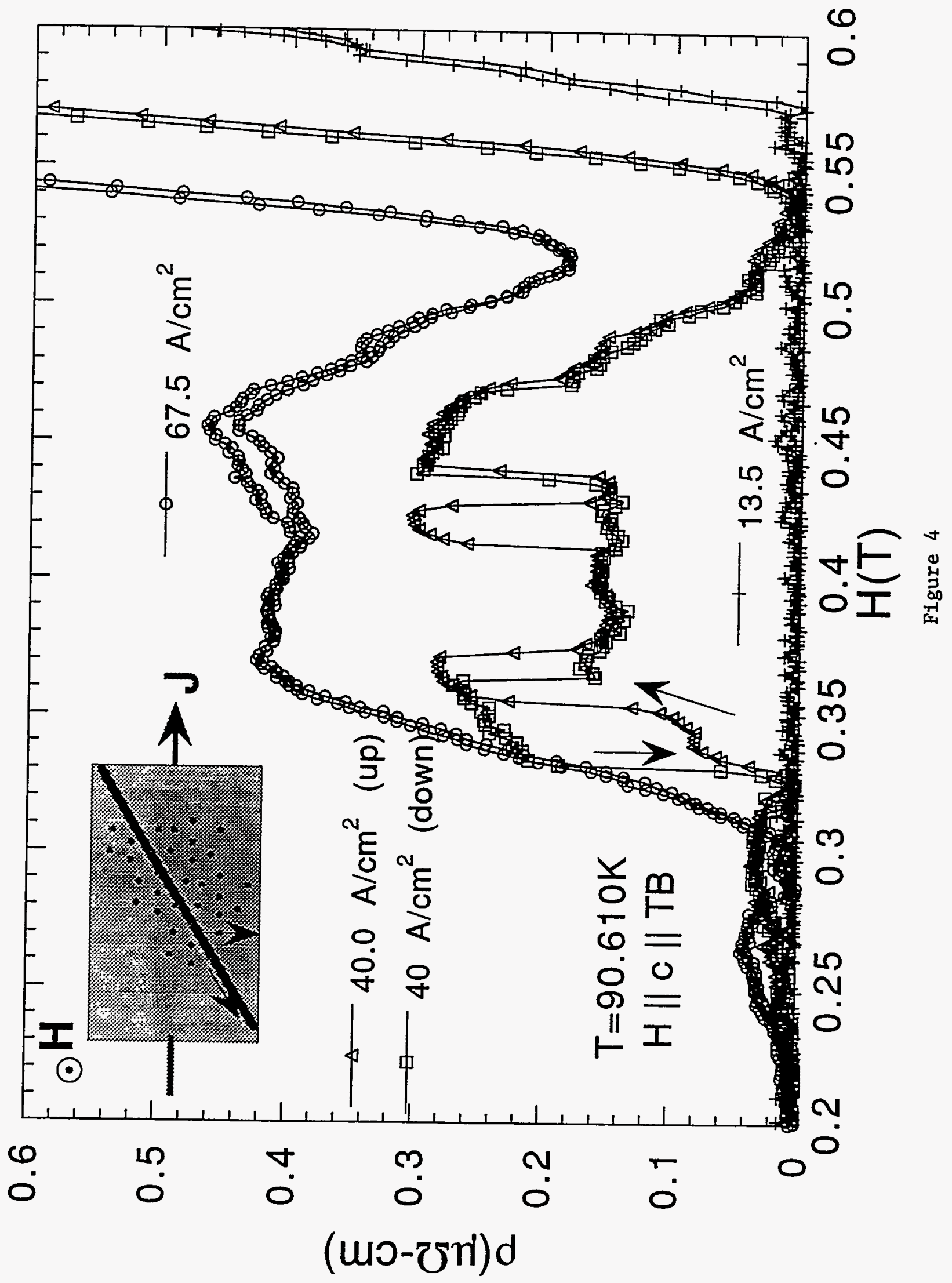

